\title{
Humanoid Prototype Development Through 3D Printing for New Technologies
}

\author{
Matheus Henrique Nunes França ${ }^{1 *}$; Fredson da Silva Oliveira ${ }^{2}$; Oberdan Rocha Pinheiro ${ }^{3}$ \\ ${ }^{1,2}$ Wyden, Engineering; ${ }^{3}$ SENAI CIMATEC, Department of Robotics: Salvador, Bahia, Brazil
}

\begin{abstract}
Robotics creates the possibility of using robots to assist humans in several tasks, especially in hostile environments to humans. In order to cope with different types of environments, it is of great importance that robots are adaptable to the environment in which they operate. So, this study aimed is to develop a prototype of a humanoid robot by $3 \mathrm{D}$ printing as close as possible to the human being. The prototype will be used as the cradle of tests and thus will advance the most diverse techniques in robotics and artificial intelligence.
\end{abstract}

Keywords: Humanoid. 3D Printing. Robotics.

The growing interest in humanoid robots has substantially helped to increase their development over the past decade. The existing humanoids can be classified as wheeled humanoids, legged humanoids, and human-like [1]. This research project offers an open-source humanoid model, printed in 3D. In particular, the use of 3D printing technologies and rapid prototyping is a central aspect of this project and makes it easy and fast, reproducing the platform, and also exploring its use in science and education. The project was initially designed with a scientific objective, aiming to be an experimental platform, opening the possibility to systematically study the role of morphology in motor sensor control in humanrobot interaction and cognitive development.

\section{Methods}

The conception of the humanoid robot takes into account all human anthropomorphic characteristics. In this sense, it was built with hybrid performance, designed to assist the motors aiming to improve energy efficiency when moving their joints. The design was developed

$\overline{\text { Received on } 18 \text { October 2019; revised } 23 \text { September } 2019 .}$ Address for correspondence: Dr. Matheus Henrique Nunes França. Avenida Orlando Gomes, N. 1845, Piatã. Zip Code: 41650-010. Salvador, Bahia, Brazil. E-mail: br_matheus@ hotmail.com. This study was selected from the V International Symposium of Innovation and Technology - SIINTEC (October 2019).

J Bioeng. Biotech. Appl. Health 2019;2(4):123-129. (C) 2019 by SENAI CIMATEC. All rights reserved. with the aid of Fusion 360 software, while the parts were manufactured using a $3 \mathrm{D}$ printer. Seventeen LOBOT LX-16A servo motors, an Xbox 360 Kinect Sensor, a raspberry pi 3, 7 mpu6050 gyros, and 8 Sparkfun 5" force sensors were used, which are the elements that comprise the prototype. Following is a description of the steps for building the prototype.

\section{Active and Passive Action}

One of the significant challenges of the project was to develop human-like walking and sturdiness when in the presence of disturbances, such as variations' terrain [2]. The main problems associated with the analysis and control of bipedal robots are changes due to the impact of the leg on the ground. These issues required a sturdy structure that minimizes engine stress. Two categories of biped robots were analyzed: 1) Operating performance: Biped robots that have feet and actuators in some of the joints (the performance is made in such a way as to take advantage of the natural characteristics of walking); and 2) Passive performance: Purely passive biped robots without feet and torque or position control.

\section{$\underline{\text { Structure Dimensioning and Servo Motors }}$ Structure}

The robotic structure has actuators that give them diverse degrees of freedom [3] needed for natural motion. For each joint, a motion study was needed to know how many engines would be inserted. For each GDL, an actuator is required; 
thus, the structure sizing depends on how many motors will be inserted. However, to determine the motors, it is necessary to have parameters such as the weight and size of the structure. It follows that the design of the structure and actuators are directly related, so we chose to make a constraint in which to make decisions. The delimitation of the structure was made, choosing a small structure, but not losing the characteristics of the human being. So, the maximum dimension of 1 meter was chosen. After the choice, it was only necessary to make a scale with the human body and then get the dimensions necessary to start the project. With the delimitations made in the structure, the engine could start being detailed.

\section{Servo Motors}

The servo (actuator) chosen for the project works with a servo mechanism that uses position feedback to control the speed and final position of the motor. Internally, a servo combines a motor with a feedback circuit, a controller, and other complementary circuits. It uses an encoder or speed sensor (encoder) that has the function of providing speed and position feedback. The servo chosen was the lobot LewanSoul LX16-A, which has as parameters the speed and position feedbacks already mentioned, as well as temperature and voltage for greater control, and also a torque and a small dimension.

\section{Computer Assisted Drawing(CAD) and Structural} Analysis

A complete study of the human body was necessary. The technic design, which was part of the project, started with the sizing of the structure and the chosen engine. The design was done in the Fusion 360 software. For the freedom of movement tests that the body would have, we corrected all errors. The model was used for production in the $3 \mathrm{D}$ printer. After the design finished, it was processed by the $3 \mathrm{D}$ printer program, in which case Simplify 3D generated the machine code ( $\mathrm{g}$ code), and, thus, made the part itself.
Foot

The project was started by simplifying the toes because of their complexity, but not compromising the march, so the foot only needed one axis of rotation for the toes. The feet (Figure 1) also included a set of force sensors for ground impact analysis and a sensor (mpu6050) containing gyroscope, accelerometer, and temperature capture. Rubber soles were used to avoid slipping on smooth surfaces. It was necessary to insert four cavities for the coupling of force sensors and a mechanical limit switch on the finger so that it did not exceed $45^{\circ}$ hyperextension.

\section{Ankle}

One actuator coupled to the foot, one to the ankle, and the other just above, to the shin to accommodate the three-foot movement actuators allowing to maximize gaps (Figure 2).

\section{Shins}

The shin (Figure 3) accommodated both the foot and knee movement motor, which resulted in space optimization in the humanoid. Other gyroscope was also placed on this limb for movement analysis, and we also inserted tubes to stiffen the structure.

\section{Knee}

In the knee, only a degree of freedom was needed; thus, the system was simple than in the ankle. As already mentioned, the engine was housed in the shin. Its transmission was made directly on the motor shaft, which maintains a ratio of 1:1 not to change its torque or speed. Despite its simplicity, the knee is a joint much demanded by the robot's structure (i.e. [4]). The structure, like the toe, also has a mechanical limit switch that limits the structure to its flexion, leaving aside unnecessary hyperextension.

\section{Thigh}

The thigh (Figure 4) was divided into two parts, the first part coupled to the knee including the 
Figure 1. a) Top view of the foot with silicone (mechanical end of stroke). b) Bottom view of the foot without silicone (showing cavities for sensors).

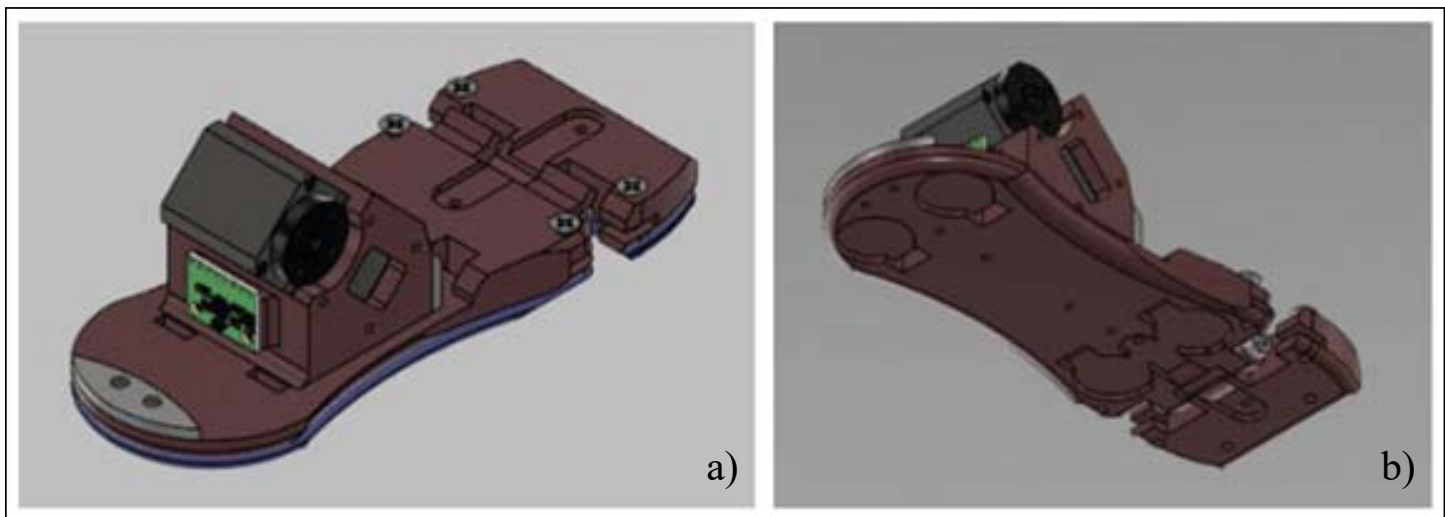

Figure 2. a) Ankle front view. b) Ankle connected to the foot.

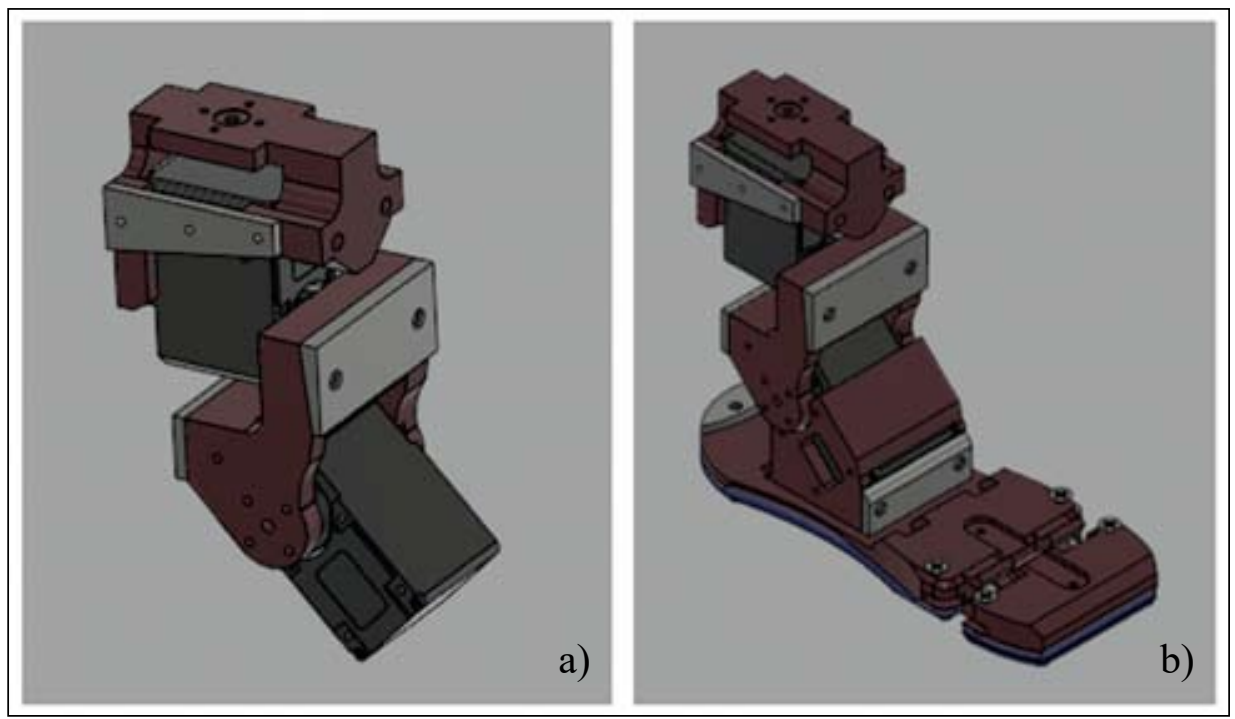

gyro sensor, structural fortification tubes, and the lateral and medial rotation motor of the hip or the thigh. The second part comprised the hip ligament that makes the abduction and hyperabduction movements.

\section{Hip}

The hip (Figure 5) must have 3 degrees of freedom for the leg and 1 degree of freedom for the trunk.

The motor shafts are connected to the structure in a cascade, which, although not anthropomorphically correct, does not affect its expected final movement. Since this is a large part of the robot's structure, it had to be divided into three parts. The first had the trunk rotation motor, and the other two connected the central part with the thigh joint. Cylinders were also placed to increase its strength and a bearing to aid in body torso movement.

\section{Body Torso}

The body torso (Figure 6) contained 3 degrees of freedom, and like the other joints of 
Figure 3. a) Front view of the shin already attached to the knee. b) Back view of the shin.

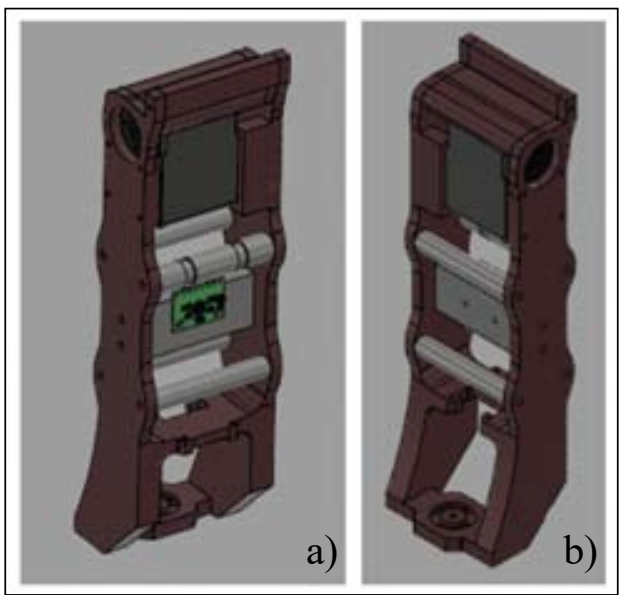

Figure 4. Thigh with all parts assembled in fusion 360 software.

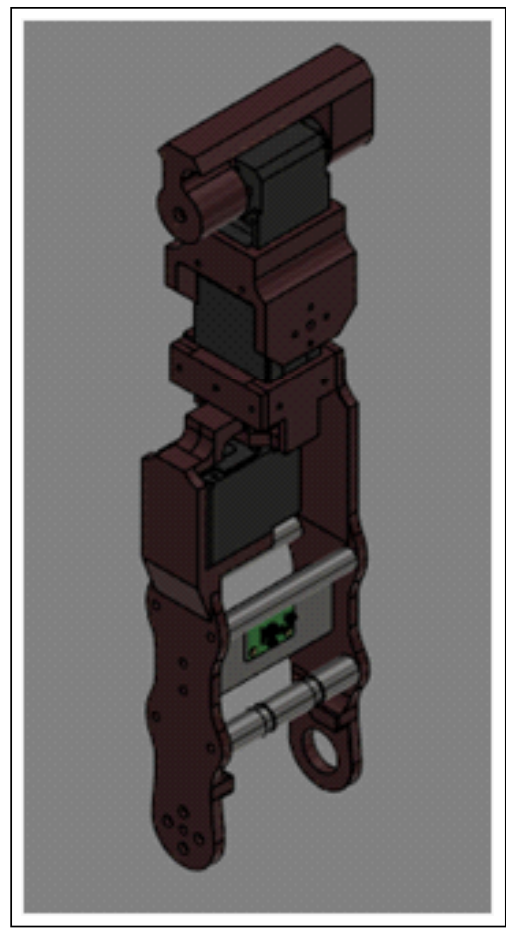

Figure 5. a) Hip in front view. b) Hip in back view with servo showing.

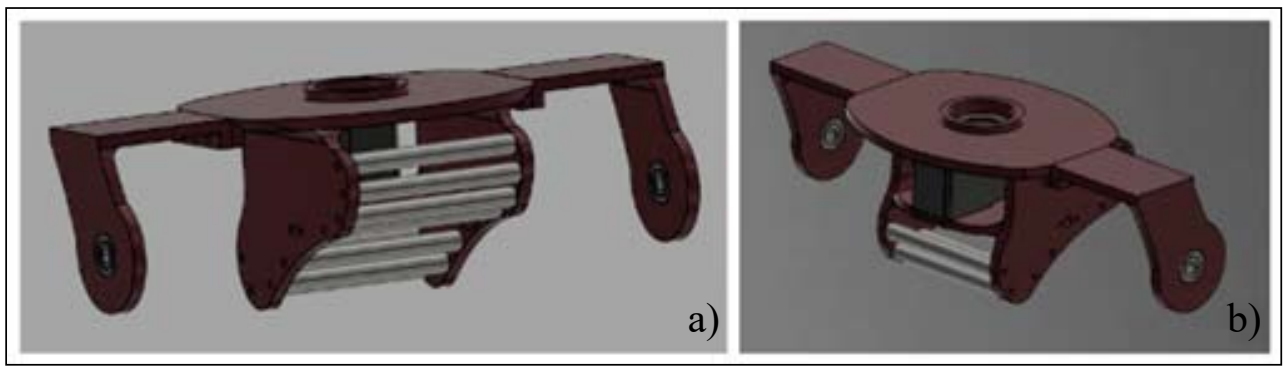


the structure, it was connected in cascade. The rotational movement was inserted into the hip. The body torso accommodated the central part of the electronics. It had a gyroscope, the buslinker card for the servo, the raspberry pi 3 for the control part, and contained speakers, an LCD screen, and a Kinect on top.

\section{Structure Production}

A very satisfactory solution was designed after thehumanoiddrawings weremade, accommodating a total of 19 degrees of freedom ( 2 of them purely passive, Table 1) and the dimensions of a human being approximately 1 meter high. This solution meets all the requirements imposed at the beginning of this study regarding measurement and handling.

Table 1. Distribution of DoF's (Degrees of Freedom) of Adam's Humanoid Robot.

\begin{tabular}{lc}
\hline Joint & DoF's Number \\
\hline Foot & $1(\mathrm{x} 2)$ \\
Ankle & $3(\mathrm{x} 2)$ \\
Knee & $1(\mathrm{x} 2)$ \\
Hip & $3(\mathrm{x} 2)$ \\
Body Torso & 3 \\
Total & 19 \\
\hline
\end{tabular}

For the construction of this structure, the 3D Anet A8 printer was used because it has a low production cost and an excellent technical quality. It used the filament petg xt, which is a material of high mechanical, chemical and temperature resistance, hardness, and neutral odor of processing. The PETG is a durable copolyester. The PET, think of bottle plastic, and G of glycol modified for extra durability. We began printing the parts by the foot, which consisted of two parts and the four parts that composed the silicone mold, followed by all the other parts that made up the right leg and the hip, only then started the left leg and the body torso. After finishing the 3D printing and the assembly, we started to place the passive actuators, and, in this case, rubber bands were used. Then, the sensors and all the electronics were placed, and just then, the plates covering the entire structure were installed.

\section{Results and Discussion}

The structural design (Figure 7) was developed in a 3D printer with an environmentally friendly material, initially built the part of the robot's body torso and legs. In future work, the upper limbs and the head will be structured. All movements have reached the required amplitudes for the desired structure as well as the desired size. The passive

Figure 6. a) Body torso with lid containing the LCD. b) Trunk with part of the visible electronics.

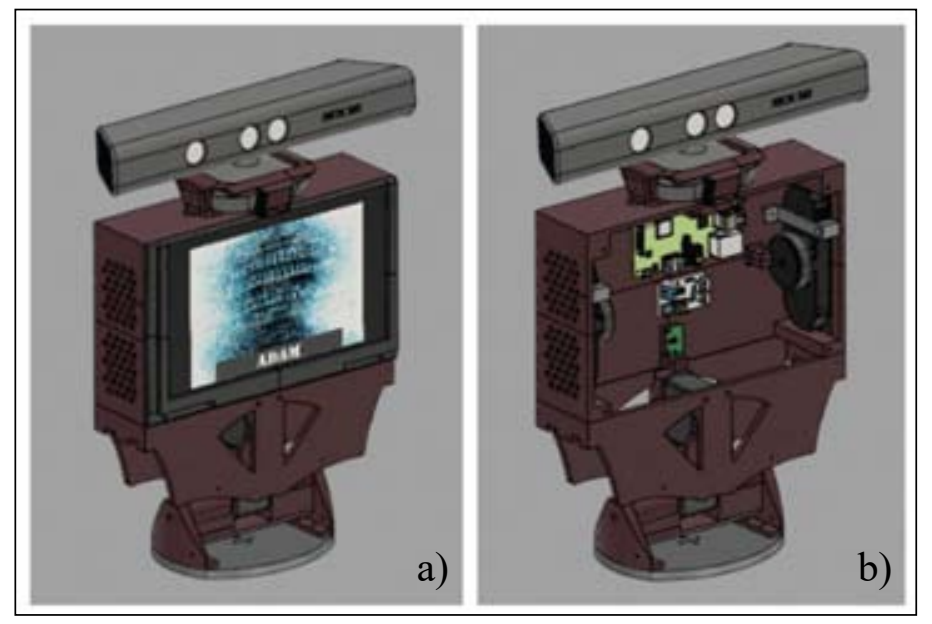


actuators that assist the servo motors have been assertivelyinserted.Amechanicalloss wasobserved in the most demanded actuator connections due to the structure is made of copolyester and not of a stronger material such as aluminum. This results in much faster wear on the movement axes, causing a lag in movement angles.

\section{Conclusion}

Robotics has been driven by the possibilities of using robots to assist humans in several tasks. In order to cope with different types of environments, it is of great importance that robots can develop mobility strategies that are adaptable to the environment in which they will operate. In this sense, our humanoid prototype aimed to generate a cradle of tests in which researchers in the field of robotics can develop their studies, thus bringing an enriching possibility of knowledge sharing, and accelerating the development in this area of this field. Another possibility for humanoids is the insertion in schools in order to increase students' interest in this area.

At first, a platform was created, which consisted of legs, body torso, and the computer vision part with Kinect. Every prototype made in a 3D printer uses both active acting (with motors) and passive acting (rubber bands) in their joints. We also used sensors to capture maximum feedback from the outside world. As future research work, the development of intelligent deliberative agent-

Figure 7. a) View without protection plates. b) View with protective plates. c) The structure already machined and with passive actuator.

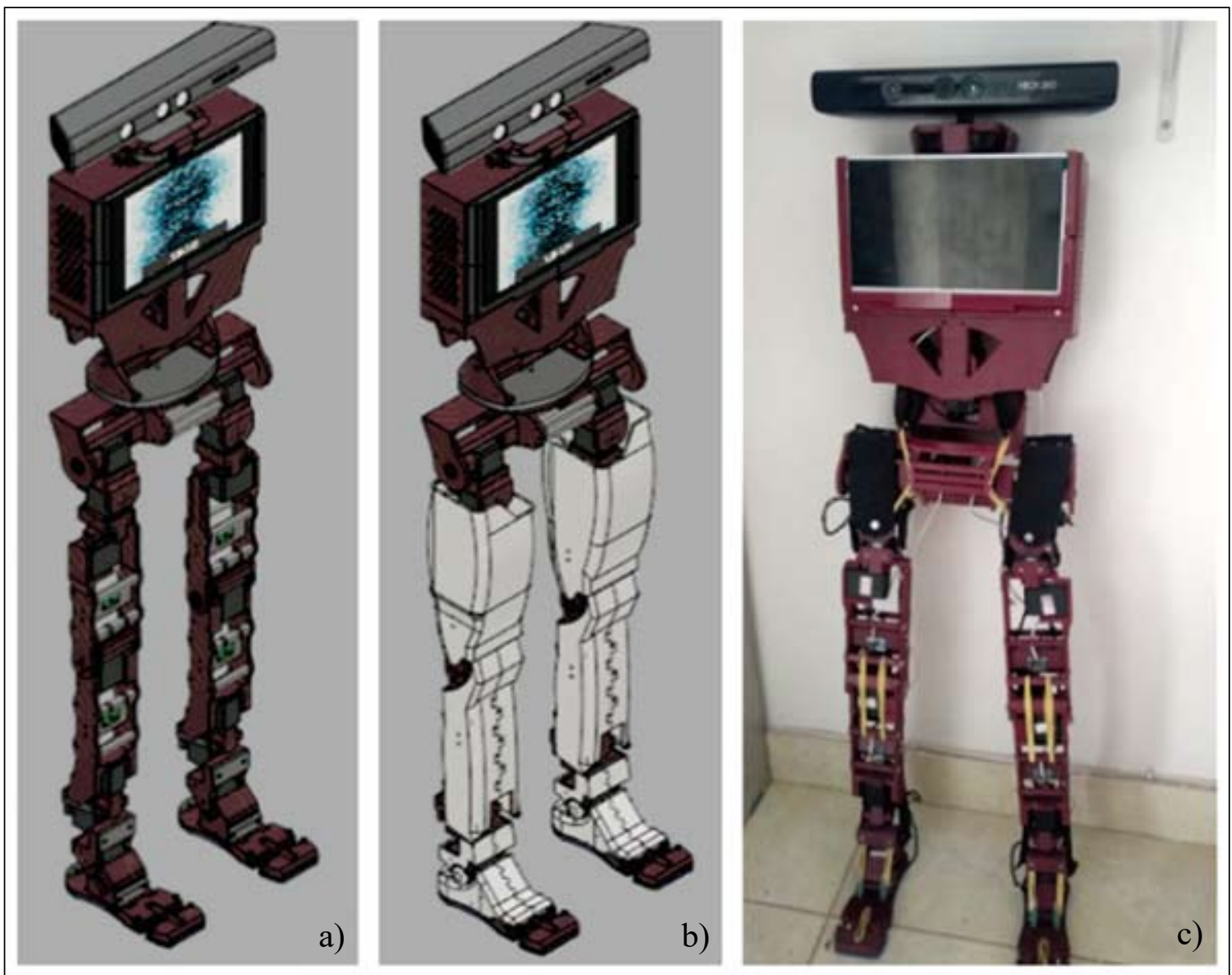


based stability and walking control using advanced machine learning techniques is suggested.

\section{References}

1. Bar-Cohen $\mathrm{Y}$ et al. The Coming Robot Revolution; Springer: Berlin, Germany; Heidelberg, Germany, 2009.

2. Sariyildiz E, Temeltas H. Turkish Journal of
Electrical Engineering \& Computer Sciences. 2017;25(2):1495-2.

3. Lida F, Minekawa Y, Rummel J, Seyfarth A. Toward a human-like biped robot with compliant legs. Robotics and Autonomous Systems. 2009;57:139144.

4. Lahr DF, Yi H, Hong D. Advanced Robotics. 2016;30(2):109-118. 